NOTES AND CORRESPONDENCE

\title{
The Applicability of the Piecewise Linear Current Profile in the Baroclinic Instability Problem
}

\author{
By Jae Min Hyun* \\ ES 82, Fluid Mechanics Branch, Space Science Laboratory, \\ Marshall Space Flight Center, Huntsville, Alabama 35812, U.S.A. \\ (Manuscript received 15 April 1980, in revised form 22 August 1980)
}

\begin{abstract}
The applicability of the piecewise linear function in place of a similar smoothly-varying current profile is examined in the baroclinic context. Within the framework of smallperturbation linearization, the behavior of the vertical velocity and the horizontal divergence is analyzed at the discontinuity of the current shear. In the conventional geostrophic-type instability regime, the discontinuity in the horizontal divergence at the shear discontinuity is suppressed, and, therefore, the piecewise linear profile leads to a useful approximation to the true solution. In the symmetric-type instability regime, however, due to the magnified discontinuity in the horizontal divergence at the shear discontinuity, the solution thus cbtained will show a major distortion, rendering the piecewise linear profile inadequate for modeling the smoothly-varying current profile. Using exemplary current profiles, numerical results are presented to demonstrate the behavior of the horizontal divergence near the discontinuity of current shear.
\end{abstract}

\section{Introduction}

In the study of stability properties of a baroclinic zonal current $U(z)$, explicit solutions of the eigenvalue problem are often difficult to find for a general current profile. As an approximation to similar smoothly-varying profiles, one may wish to use a piecewise linear function profile in the baroclinic studies, in line with the practice in some other branches of hydrodynamic stability problems. The baroclinic instabilities of the simple smoothly-varying profile $U \sim z$ in various parameter ranges have been extensively investigated (e.g., Stone, 1966, 1970; Tokioka, 1970). If the use of the piecewise linear profile can be justified, one can solve the eigenvalue equation for $U \sim z$ within each section, and then join them up by means of the matching conditions at the section interface at which the basic state shear $d U / d z$ has a jump. The purpose of this paper is to examine the effect of the use of

\footnotetext{
* NRC Senior Postdoctoral Research Associate.
}

the piecewise linear profile as an approximation to a similar smoothly-varying profile on the linearized baroclinic instabilities.

Some important difference has to be noted between the matching conditions at the section interface in the present context and the physical interface conditions in the quasi-geostrophic layer model of Pedlosky (1964). In the latter, the layer interface is physical in the sense that it separates two layers of homogeneous fluids. Both the density and the zonal current speed are assumed to be uniform but different in each layer in Pedlosky's model. In the present case, the term interface is employed to merely denote the level $z=z_{0}$ at which the basic state shear $d U / d z$ has a discontinuity. In this connection, the present piecewise linear current profile model should also be distinguished from the multi-level finite-difference model (e.g., Hirota, 1968).

A common form of the linearized governing differential equation when $U \sim z$ is derived with the vertical velocity as the dependent variable (e.g., Stone, 1966; Tokioka, 1970). Obviously, 
both $w$, the vertical velocity, and $\partial w / \partial z$, which can be interpreted as the horizontal divergence for a Boussinesq fluid, are continuous at all levels $z$ for a smoothly-varying profile $U(z)$. In this paper, under the assumption of small-perturbation linearized normal-mode disturbances, we shall examine how the otherwise continuous nature of $w$ and $\partial w / \partial z$ is affected as the smoothly-varying profile $U(z)$ is replaced by a piecewise linear function. Based on the physical requirement that both the normal velocity and the pressure be continuous at the material fluid interface (Drazin and Howard, 1966), we shall derive the matching conditions, expressed in terms of $w$ and $\partial w / \partial z$, at the discontinuity of $d U / d z$. The behavior of $w$ and $\partial w / \partial z$ at the section interface, i.e., at the level of $d U / d z$ discontinuity, will guide us to assess the applicability of the piecewise linear profile as an approximation to the actual smoothly-varying profile for various types of perturbations (Stone, 1966) in linear baroclinic instabilities.

\section{Derivation of the matching conditions}

We consider the small-amplitude linearized normal-mode perturbations to a thermal wind basic state in a Boussinesq fluid on an $f$-plane. Using standard meteorological notation, in a given section the basic state zonal current $U$ can be specified as

$$
U=\left(\frac{d U}{d z}\right) z,\left(\frac{d U}{d z}\right) \text { is constant. }
$$

The perturbations of this flow are assumed to be small and to have time and space dependences of the form

$$
\phi=\hat{\phi}(z) \exp \{i(k x+l y-k c t)\} .
$$

Superposing the small perturbations to the basic state, and the linearized system of equations may be reduced to a single eigenvalue differential equation for the vertical velocity $w$ [see Tokioka, 1970, Eq. 2-20]:

$$
\begin{aligned}
& {\left[k^{2}(U-c)^{3}-f^{2}(U-c)\right] \frac{d^{2} \hat{w}}{d z^{2}}} \\
& \quad+\left[2 f^{2}-2 i l f(U-c)\right]\left(\frac{d U}{d z}\right) \frac{d \hat{w}}{d z} \\
& \quad+M(U-c)\left\{g S-2 i\left(\frac{d U}{d z}\right)^{2} l f /[M(U-c)]\right\} \\
& \quad \times \hat{w}=0 .
\end{aligned}
$$

where $M \equiv k^{2}+l^{2}$, and $S$ is the vertical gradient of the basic potential temperature distribution. [The properly nondimensionalized version of Eq
(3) is given in Stone (1966, Eq. 1.2).] Notice that Eq. (3) was derived only under the assumption of the small-amplitude linearization, and Eq. (3) is not a consequence of the Rossby number expansion as was done in some other models (e.g., Pedlosky, 1964).

In order to match the solutions of Eq. (3) at the discontinuity of $d U / d z$, the interface condition is based on the continuity of the normal velocity and the pressure at the material fluid interface. Since $U$ itself is continuous, the kinematic matching condition is straightforward; the vertical velocity is continuous at the discontinuity of $d U / d z$.

For the dynamic matching condition, we see that the perturbation momentum equation in the $x$ direction is [Eq. (2-9) of Tokioka, 1970]

$$
i k(U-c) \hat{u}+(d U / d z) \hat{w}-f \hat{v}=-i k \hat{p},
$$

where $p$ is the deviation from hydrostatic pressure divided by a mean density.

In general, the dynamic matching condition calls for the continuity of pressure at the material fluid interface with mean position, say at $z=z_{0}$ (Drazin and Howard, 1966). Therefore, under the assumption of small-amplitude linearization, to first degree in the perturbations, we have from Eq. (4)

$$
\left[i k(U-c) \hat{u}+\left(\begin{array}{c}
d U \\
d z
\end{array}\right) \hat{w}-f \hat{v}\right] \begin{aligned}
& z_{0}+\varepsilon \\
& z_{0}-\varepsilon
\end{aligned}=0,
$$

at $z=z_{0}$, where brackets denote the jump of the contents at the interface. Again, the application of the pressure continuity at the mean level $z=z_{0}$ in Eq. (5) instead of the actual disturbed surface is consistent with the linearization concept, and this practice should not be confused with the Rossby number expansion procedure.

It is clear from Eq. (5) that in order to obtain the dynamic matching condition suitable for the governing equation (3), we need to express the horizontal velocity components $(\hat{u}, \hat{v})$ in terms of $\hat{w}$ and $d \hat{w} / d z$. Following the developments of Tokioka (1970), retaining only the linear terms, we have for $(\hat{u}, \hat{v})$, after considerable algebraic manipulation:

$$
\begin{gathered}
\hat{u}=\frac{i k(d \hat{w} / d z)}{M}+\frac{i f l \frac{d \hat{w}}{d z}-l^{2} \frac{d U}{d z} \hat{w}}{i k M(U-c)}, \\
\hat{v}=-\left\{\frac{i l(d U / d z)}{M(U-c)}\right\} \hat{w}+\left\{\frac{i l(U-c) f}{M(U-c)}\right\} \frac{d \hat{w}}{d z}
\end{gathered}
$$

Substituting Eqs. (6) and (7) into Eq. (5), the interface jump can be shown to be 


$$
\begin{aligned}
& {\left[\left\{\frac{f^{2}-k^{2}(U-c)^{2}}{M(U-c)}\right\} \frac{d \hat{w}}{d z}\right.} \\
& \left.\quad+\left\{\frac{i f l+k^{2}(U-c)}{M(U-c)}\right\} \frac{d U}{d z} \hat{w}\right]_{z_{0}-\varepsilon}^{z_{0}+\varepsilon}=0 .
\end{aligned}
$$

This interface jump condition can also be obtained by integrating the differential equation for $\hat{w}$ with a general $U(z)$ over a small interval across $z=z_{0}$.

In view of the continuity of $U$, and subsequently the continuity of $\hat{w}$ owing to the kinematic matching requirement, at $z=z_{0}$, Eq. (8) indicates that $d \hat{w} / d z$ is in general discontinuous at the discontinuity of $d U / d z$.

It is now advantageous to rewrite Eq. (8) as

$$
\begin{aligned}
& {\left[\left\{1-k^{* 2}\left(U_{0}^{*}-c^{*}\right)^{2}\right\} \frac{d w^{*}}{d z^{*}}\right.} \\
& \left.\quad+\left\{k^{* 2}\left(U_{0} *-c^{*}\right)+i l^{*}\right\} \frac{d U^{*}}{d z^{*}} w_{0} *\right]_{z_{0}^{*}-\varepsilon}^{z_{0}^{*}+\varepsilon} \\
& \quad=0 .
\end{aligned}
$$

where the quantities are nondimensionalized as

$$
\begin{aligned}
& \left(k^{*}, l^{*}\right)=(k \Delta U / f, l \Delta U / f), \\
& \left(U^{*}, c^{*}, w^{*}\right)=(U, c, \hat{w}) / \Delta U . \\
& z^{*}=z / D .
\end{aligned}
$$

In Eq. (9) the subscript 0 denotes the values at $z^{*}=z_{0}{ }^{*}$, and $\Delta U$ and $D$ are the characteristic velocity scale and the vertical length scale, respectively.

\section{Discussion}

Noting the continuity of $U$ and $w^{*}$, and after rearranging terms, one can obtain from Eq. (9) an explicit expression relating to the discontinuity in $d w^{*} / d z^{*}$ at the interface of shear discontinuity:

$$
\frac{d w^{*}}{d z^{*}}-\frac{d w^{*}}{d z^{*}}=\left\{\frac{\left[k^{* 2}\left(U_{0}^{*}-c^{*}\right)+i l^{*}\right] w_{0}^{*}}{k^{*}\left(U_{0} *-c^{*}\right)^{2}-1}\right\} V,
$$

where

$$
\Lambda \equiv \frac{d U^{*}}{d z^{*}}-\frac{d U^{*}}{d z^{*}}
$$

Obviously, for a smoothly-varying current profile $\Lambda=0$ at all levels, and the horizontal divergence $d w^{*} / d z^{*}$, as well as the vertical velocity $w^{*}$, is continuous at all levels. We intend here to peruse the implications of the dynamic matching requirement Eq. (10), and thereby we shall be able to assess the applicability of the piecewise linear function model to the various types of baroclinic instabilities classified by Stone (1966).

The principal results of Stone's investigation of the nongeostrophic effects on the baroclinic stability problem reveal three basically different types of instabilities. The largest growth rates are associated with (1) the conventional geostrophic-type instability $\left(k^{*}\right.$ small, $\left.l^{*} \rightarrow 0\right)$ for large Richardson number, (2) the symmetric-type instability $\left(k^{*} \rightarrow 0, l^{*} \gg 1\right)$ for the Richardson number less than 1, (3) the Kelvin-Helmholtz-type instability $\left(k^{*} \gg 1, l^{*} \rightarrow 0\right)$ for the Richardson number less than $1 / 4$.

In the first, in the conventional geostrophictype instability regime $\left(k^{*}\right.$ small, $\left.l^{*} \rightarrow 0 ; R_{i} \gg 1\right)$ setting $c^{*} \sim 1$, then the linearized matching condition Eq. (10) yields

$$
\begin{aligned}
& \frac{d w^{*}}{d z^{*}}+-\frac{d w^{*}}{d z^{*}}- \\
& =-\left\{k^{* 2}\left(U_{0}^{*} c^{*}\right)\right\}\left\{1+k^{* 2}\left(U_{0}^{*}-c^{*}\right)^{2}\right\} w_{0}^{*} \Lambda .
\end{aligned}
$$

which implies that

$$
\frac{d w^{*}}{d z^{*}}+-\frac{d w^{2}}{d z^{*}}=0\left(k^{* 2}\right) \Lambda .
$$

Eq. (12) shows that in the geostrophic-type instability regime, the jump in the horizontal divergence corresponding to the jump in the current shear is at most $O\left(k^{* 2}\right)$. Therefore, to the leading order

$$
\frac{d w^{*}}{d z^{*}}+-\frac{d w^{*}}{d z^{*}}=0 \text {. }
$$

pointing to the continuity of the horizontal divergence at the discontinuity of $d U / d z$ in the quasigeostrophic situations. In fact, the relation in Eq. (13) can also be derived by considering the linearized version of the quasi-geostrophic vorticity equation (dimensional)

$$
\left(\frac{\partial}{\partial t}+U \frac{\partial}{\partial x}\right) \nabla^{2} \phi=f \frac{\partial w}{\partial z},
$$

where $\phi$ is the perturbation quasi-geostrophic streamfunction. Assuming normal mode disturbances, Eq. (14) yields [e.g., see Tang, 1975, Eq. 4a]

$$
(U-c) \hat{\psi}=-\frac{f}{i k M} \frac{d \hat{w}}{d z} .
$$

Here the continuity of the pressure, hence the continuity of the quasi-geostrophic streamfunction $\psi$, and the continuity of $U$ lead to the continuity of $d w / d z$. In summary, in the geostrophic-type instability regime, as manifested by the matching condition in Eq. (12), the artificiality due to the use of the piecewise linear profile is greatly suppressed to cause only an $O\left(k^{* 2}\right)$ dis- 
continuity of the horizontal divergence at the level of $d U / d z$ discontinuity. The above analysis provides justification to the usual practice of adopting the piecewise linear profile, together with the matching condition in Eq. (13), in the study of quasi-geostrophic perturbations in the multi-layer Eady-like model (e.g., Tang, 1975).

Secondly, let us specialize the linearized matching condition Eq. (10) to the symmetric-type instability regime $\left(k^{*} \rightarrow 0, l^{*} \gg 1 ; R_{i}<1\right)$. Following Stone, we set the growth rate $k^{*} c^{*} \sim 1$ for this case, and Eq. (10) reduces to

$$
\left.\frac{d w^{*}}{d z^{*}}\right|_{+}-\left.\frac{d w^{*}}{d z^{*}}\right|_{-} \cong\left\{\frac{i l^{*}}{k^{* 2}\left(U_{0}^{*}-c^{*}\right)^{2}-1}\right\} w_{0}^{*} \Lambda .
$$

It is seen in Eq. (16) that the discontinuity of the current shear, $\Lambda$, is greatly magnified $\left(l^{*} \geqslant 1\right)$ to result in a large discontinuity in the horizontal divergence $d w^{*} / d z^{*}$. In other words, because of the small meridional scale $\left(l^{*} \gg 1\right)$, a (presumably small) discontinuity in the current shear introduced by the use of a piecewise linear profilc will give rise to a greatly amplified discontinuity in the horizontal divergence at the section boundary. This amplified discontinuity of the horizontal divergence at the discontinuity of $d U / d z$ represents a major distortion of the solution, particularly near the section boundaries, compared to the true continuous behavior of the horizontal divergence at all levels for the smoothly-varying actual current profile. Therefore, one can conclude that, in contrast to the case of conventional geostrophic-type instability regime discussed earlier, the attempt to replace the actual smoothly-varying current profile by a piecewise linear function in the symmetric-type instability regime does not seem to be fully justified.

Finally, we consider the case of the Helmholtztype instability regime $\left(k^{*} \gg 1, l^{*} \rightarrow 0 ; R_{i}<1 / 4\right)$. Again, following Stone, we set $c^{*} \sim 1$, then the linearized matching condition Eq. (10) shows

$$
\left.\frac{d w^{*}}{d z^{*}}\right|_{+}-\frac{d w^{*}}{d z^{*}} \cong\left(\frac{1}{U_{0}^{*}-c^{*}}\right) w_{0}^{*} \Lambda \text {. }
$$

If written in dimensional form, Eq. (17) becomes

$$
\text { , }\left.\frac{d \hat{w}}{d z}\right|_{+}-\left.\frac{d \hat{w}}{d z}\right|_{-} \cong\left(\frac{\hat{w}_{0}}{U_{0}-c}\right)\left(\left.\frac{d U}{d z}\right|_{+}-\frac{d U}{d z} ;\right)
$$

which can readily be recognized to be the matching condition for the non-rotating shear flow problem (Drazin and Howard, 1966, Eq. 2-13). Clearly, in this case the Rossby number of the disturbance is large $\left(k^{*} \gg 1\right)$ that the rotational effect, represented by the Coriolis force term in the momentum equation, is neglected in comparison to the inertia term, and the problem reduces essentially to a non-rotating case. The use of the piecewise linear profile in the (nonrotating) shear flow problem has been treated elsewhere (e.g., Drazin and Howard, 1966), and this case is of less interest in the present baroclinic context.

\section{Examples}

In order to demonstrate the behavior of the eigenfunction when the piecewise linear profile is employed, numerical computations were performed for model current profiles. As shown in Fig. 1, as the exemplary smoothly-varying profile (labeled $S$ ) we take Eady's (1949) model current

$$
U(z)=(\Delta U / D) z .
$$

The piecewise linear profile (labeled P) can be described as

$$
U(z)= \begin{cases}\left(\Delta U_{1} / H_{1}\right) z & 0 \leq z \leq H_{1} \\ \Delta U_{1}+\left(\Delta U_{2} / H_{2}\right)\left(z-H_{1}\right) & H_{1} \leq z \leq D\end{cases}
$$

In the ensuing discussion, these current profiles will be made dimensionless (and denoted by the asterisk) using the velocity scale $\Delta U$ and the vertical length scale $D$. For simplicity, the Brunt-Väisälä frequency $N$ is assumed to be constant throughout the entire fluid depth $0 \leq$ $z^{*} \leq 1$. Consequently, the overall Richardson number is defined $R_{i}=N^{2} /(\Delta U / D)^{2}$.

Due to the nondimensionalization scheme adopted, the dimensionless shear for the smoothlyvarying profile of Eq. (19) is unity. In the case of the piecewise linear profile of Eq. (20), the dimensionless jump in current shear, $\Lambda$, at the

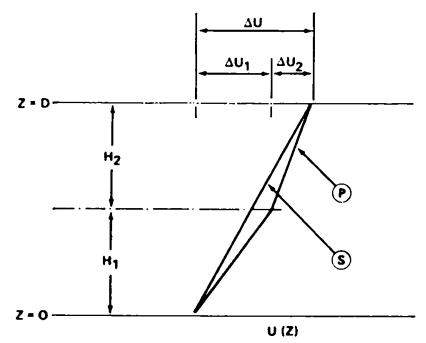

Fig. 1 The exemplary zonal current profiles. (S) denotes the smoothly-varying profile and $D$ the piecewise linear profile. 
interface $z^{*}=H_{1} *$ is

$$
\begin{aligned}
\Lambda & =\frac{\Delta U_{2} *}{H_{2} *}-\frac{\Delta U_{1} *}{H_{1} *} \\
& =\frac{1-\Delta U_{1} *}{1-H_{1} *}-\frac{\Delta U_{1} *}{H_{1} *} .
\end{aligned}
$$

The numerical technique used to solve the eigenvalue problem is the "shooting method". With an educated guess for the eigenvalue $c^{*}$, the differential equation for the prescribed current profile $U^{*}\left(z^{*}\right)$ is integrated from the lower boundary $z^{*}=0$ to the interface $z^{*}=H_{1}{ }^{*}$. The interface jump condition, Eq. (10), is then incorporated, and integration continues until the upper boundary $z^{*}=1$ is reached. The technique is to repeat the above procedure until we find the right eigenvalue $c^{*}$ so that the boundary conditions at both $z^{*}=0$ and $z^{*}=1$ are satisfied. The algorithm used by Wiin-Nielsen (1971) and Hyun and Peskin (1978) was adopted for the present problem. The reader is referred to the above papers for detailed numerical methods.

First, in order to examine the effect of the piecewise linear profile in the conventional geostrophic-type instability regime, the following parameters were chosen for computation:

$$
\begin{aligned}
& R_{i}\left[=N^{2} /(\Delta U / D)^{2}\right]=2.0, \\
& k^{*}=0.14, \quad l^{*}=0.0 .
\end{aligned}
$$

Two sets of piecewise linear profiles of Eq. (20) were tested:

(a) $\Delta U_{1}^{*}=0.525, H_{1}^{*}=0.5$, thus $\Lambda=-0.10$ :

(b) $\Delta U_{1} *=0.475, H_{1}^{*}=0.5$, thus $\Lambda=0.10$.

For the smoothly-varying profile of Eq. (19), the eigenvalue is found to be $c^{*}=(0.5,0.2870)$. The computed eigenvalues are $c^{*}=(0.5130,0.2869)$ for the profile of Eq. $(22-\mathrm{a})$, and $c^{*}=(0.4857$, 0.2872 ) for the profile of Eq. (22-b). Also computed are the wave structures of the vertical velocity $w^{*}$ and the horizontal divergence $d w^{*} /$ $d z^{*}$. As can be foreseen from the analysis in Section 3, no noticeable difference was noted in the behavior of $w^{*}$ and $d w^{*} / d z^{*}$ between the cases of the smoothly-varying profile of Eq. (19) and the piecewise linear profiles of Eq. (22). The discontinuity in the horizontal divergence caused by the current shear jump of the piecewise linear profiles of Eq. (22) is sufficiently small $\left(0\left(k^{* 2}\right)\right)$ that the horizontal divergence appears to be essentially continuous at the interface. The vertical distributions of $w^{*}$ and $d w^{*} / d z^{*}$ for Eady's model current of Eq. (19) in the geo- strophic-type instability regime are well-documented (e.g., Eady, 1949; Hyun and Fowlis, 1979), and they are not reproduced here.

We now consider the more interesting case of the symmetric-type instability regime. Computations were made for the following parameters:

$R_{i}\left[=N^{2} /(\Delta U / D)^{2}\right]=0.5, \quad k^{*}=0.1, \quad l^{*}=5.0 . \quad$ Two sets of piecewise linear profiles of Eq. (20) were tested :

Two sets of piecewise linear profiles of Eq. (20) were tested:

(a) $\Delta U_{1}=0.42, \quad H_{1} *=0.40$, thus $\Lambda=-0.083$

(b) $\Delta U_{1}=0.38, H_{1} *=0.40$, thus $\Lambda=0.083$

For the smoothly-varying profile of Eq. (19), the eigenvalue is found to be $c^{*}=(0.5,1.3945)$. The computed eigenvalues are $c^{*}=(0.4947,1.3770)$ for the profile of Eq. (23-a), and $c^{*}=(0.5046$, 1.4078) for the profile of Eq. (23-b). Fig. 2 displays the wave structures of $w^{*}$ (dashed line) and $d w^{*} / d z^{*}$ (solid line) computed for the above three profiles. It is clearly seen that the dis-
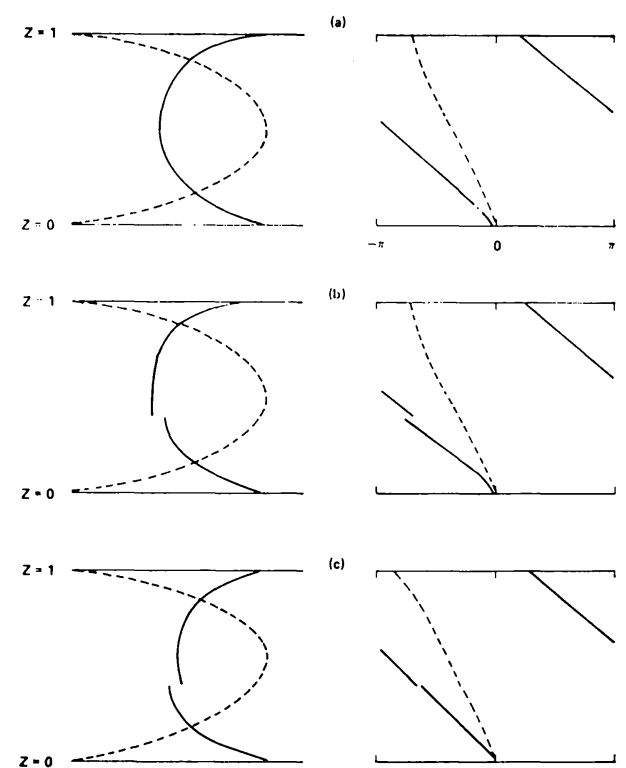

Fig. 2 Wave structures of the vertical velocity (dashed line) and the horizontal divergence (solid line) in the symmetric-type instability regime. Conditions are $R_{i}\left[=N^{2} /(\Delta U / D)^{2}\right]=$ $0.5, \quad k^{*}=0.1, \quad l^{*}=5.0$. The left diagrams show the amplitude and the right diagrams show the negative phase. The current profiles are (a) the smoothly-varying profile of Eq. (19), (b) the piecewise linear profile of Eq. (23-a), and (c) the piecewise linear profile of Eq. (23-b). 
crepancy between the horizontal divergence for the piecewise linear profile (see Fig. 2-b and Fig. 2-c) and the horizontal divergence for the similar smoothly-varying profile (see Fig. 2-a) is pronounced near the interface $z^{*}=H_{1}{ }^{*}$. As was shown in the analysis in Section 3 , due to the largeness of $l^{*}$ in the symmetric-type instability regime, a small discontinuity in current shear, $\Lambda$, gives rise to the amplified discontinuity in the horizontal divergence.

\section{Conclusions}

Based on the linearized matching requirement at the discontinuity of the current shear, an analysis has been made of the usefulness of the piecewise linear function in approximating the actual smoothly-varying current profile in the baroclinic context. In the conventional geostrophic-type instability regime, which has received most attention in the meteorological literature, the discontinuity in the horizontal divergence at the shear discontinuity is greatly suppressed, and, therefore, the piecewise linear profile leads to a useful approximation to the-true solution. In the symmetric-type instability regime, however, due to the magnificd discontinuity in the horizontal divergence at the shear discontinuity, the solution obtained for the piecewise linear profile will include a major distortion, making the piecewise linear profile inadequate for modeling the smoothly-varying current profile.

\section{Acknowledgements}

The author is grateful to Dr. W. W. Fowlis of Marshall Space Flight Center for reading the earlier version of this paper and providing helpful comments. I am grateful to NASA, Office of Space and Terrestrial Applications, for supporting my National Research Council Associate ship.

\section{References}

Drazin. P. G. and L. N. Howard, 1966: Hydrodynamic stability of parallel flow of inviscid fluid. Advances in Applied Mechanics, Vol.9, Academic Press, New York, 85 pp.

Eady, E. T., 1949: Long waves and cyclone waves. Tellus, 1, 33-52.

Hirota, I., 1968: On the dynamics of long and ultra-long waves in a baroclinic zonal current. J. Meteor. Soc. Japan, 46, 234-249.

Hyun, J. M. and W. W. Fowlis, 1979: The wave structures of the Eady model of baroclinic instability. NASA Technical Paper 1548.

—, and R. L. Peskin, 1978: Baroclinic instabilities of a deep fluid. Part II. J. Atmos. Sci., 35, 169-174.

Pedlosky, J., 1964: The stability of currents in the atmosphere and the ocean: Part I. J. Atmos. Sci., 28, 944-953.

Stone, P. H., 1966: On non-geostrophic baroclinic stability. J. Atmos. Sci., 23, 390-400.

- 1970: On non-geostrophic baroclinic stability: Part II. J. Atmos. Sci., 27, 721-726.

Tang, C.-M., 1975: Baroclinic instability of stratified shear flows in the ocean and atmosphere. $J$. Geop!lys. Res., 80, 1168-1175.

Tokioka, T., 1970: Non-geostrophic and nonhydrostatic stability of a baroclinic fluid. $J$. Meteor. Soc. Japan, 48, 503-520.

Wiin-Nielsen, A., 1971: A study of the baroclinic instability problem and the lower boundary condition. J. Geophys. Res., 27, 6497-6505.

\title{
傾圧不安定性問題で基本流を折線近似することについて
}

\author{
Jae M. Hyun
}

Marshall Space Flight Center, U.S.A. 